\title{
Autonomous leader-follower formation control of non-holonomic wheeled mobile robots by incremental path planning and sliding mode augmented tracking control
}

\author{
Kishorekumar H. Kowdiki*, \\ Ranjit Kumar Barai and Samar Bhattacharya
}

Department of Electrical Engineering,

Jadavpur University,

Kolkata 700032, West Bengal, India

Email: kowdikikh@gmail.com

Email: ranjit.k.barai@gmail.com

Email: samar.bhattacharyya@gmail.com

*Corresponding author

\begin{abstract}
This paper presents a novel robust and autonomous formation control scheme for wheeled mobile robots in the leader-follower formation control framework considering their non-holonomic constraints. In the proposed formation control scheme, the leader robot of the group plans its path of navigation autonomously in a cluttered environment by employing incremental path planning by modified artificial potential field. Then, the follower robots in the group plan their path in order to follow the leader robot by maintaining a particular formation using the separation-bearing $(l-\psi)$ control. Then the formation control problem has been transformed into a trajectory tracking control problem. The kinematic control component of the tracking controller provides the necessary velocity input for eliminating the non-holonomic constraints, whereas, the sliding mode augmented robust trajectory tracking control component minimises the effects of nonlinearities, model uncertainties, parameter variations, and disturbances. The effectiveness of the proposed control law has been established by simulation studies.
\end{abstract}

Keywords: artificial potential field; autonomous navigation; formation control; leader-follower; trajectory tracking; kinematic control; sliding mode control.

Reference to this paper should be made as follows: Kowdiki, K.H., Barai, R.K. and Bhattacharya, S. (2019) 'Autonomous leader-follower formation control of non-holonomic wheeled mobile robots by incremental path planning and sliding mode augmented tracking control', Int. J. Systems, Control and Communications, Vol. 10, No. 3, pp.191-217.

Biographical notes: Kishorekumar H. Kowdiki is a Research Scholar in Jadavpur University, Kolkata, India. He graduated from Dr. Babasaheb Ambedkar Maratwada University, Aurangabad, Maharashtra in 1994 as an Instrumentation Engineer. He received his Master's in Instrumentation and Control Engineering with specialisation in process instrumentation from University of Pune, Pune, India in 2008. He had worked in the design, erection and commissioning industry from 1994 to 1997 . Presently, he is working as an Associate Professor in Instrumentation and Control Engineering at Government College of Engineering and Research, Awasari (Khurd), Tal-Ambegaon, Dist-Pune. His current research interests include wheeled mobile robots and non-linear control. 
Ranjit Kumar Barai graduated in Bachelor of Electrical Engineering and Master of Electrical Engineering from the Jadavpur University, in 1993 and 1995 respectively. He was awarded his $\mathrm{PhD}$ in Artificial Systems Science (with specialisation in Mechatronics and Robotics) in 2007 from the Chiba University, Japan. He has performed post-doctoral research at Rolls-Royce Corporate Laboratory at the Nanyang Technological University, Singapore, from 2015 to 2016 on robotised manufacturing. He has more than 23 years of working experience in industry, research and teaching at graduate and post-graduate levels. He has supervised several Master's and $\mathrm{PhD}$ theses in the areas of mechatronics, robotics and control systems. His research interests include mechatronics, robotics, control systems, machine learning, modelling and system identification, and real-time systems. $\mathrm{He}$ is currently a Full Professor in the Control Systems Division, Department of Electrical Engineering, Jadavpur University.

Samar Bhattacharya is a former Professor and the Head of the Department of Electrical Engineering, Jadavpur University and received his graduate, post-graduate and doctoral degrees from the Jadavpur University in 1976, 1980 and 1987, respectively. He has a teaching and research experience of almost three decades. He is a highly regarded academic in the fields of control and guidance, real-time systems, safety critical systems, software audit and technology enhanced learning.

\section{Introduction}

The study on the collective behaviour of birds, animals, fishes, etc. has not only drawn the attention of biologists, but also of computer scientists and roboticists (Reynolds, 1987). Thus several methods of cooperative control (Olfati-Saber et al., 2007; Arai et al., 2002) of multi-agent system have been evolved, where a single robot is not sufficient to accomplish the given task, like navigation and foraging of unknown territory. The advantages of using a team of robots include robustness, flexibility, and adaptability to unknown dynamic environments. By formation control, we simply mean the problem of controlling the relative positions and orientations of robots in a group, while allowing the group to move as a whole. For mobile robots, two of the basic functionalities are to navigate (navigation) and to follow (tracking control).

There are roughly three approaches to multivehicle coordination reported in the literature:

1 Behaviour-based approach (Balch and Arkin, 1998), where behavioural attributes are given to the multi-agents like: to avoid-static-obstacle, to avoid-robot, to move-to-goal, to maintain-formation.

2 Leader-follower-based approach such as in Desai et al. (1998) where, two scenarios for feedback control are described. In the first scenario, one robot follows another by controlling the relative distance and orientation between the two and is popularly known as ' $(l-\psi)$ control'; and in the other scenario, a robot maintains its position in the formation by maintaining a specified distance from two robots, or from one robot and an obstacle in the environment and is popularly known as ' $(l-l)$ control'. 
3 Virtual structure-based approach (Lewis and Tan, 1997; Egerstedt and Hu, 2001; Ren and Beard, 2004), where a general control strategy is developed to force an ensemble of robots to behave as if they were particles embedded in a rigid structure. Among the above three approaches, the leader follower framework with $(l-\psi)$ method offers computational simplicity. Hence, it is employed in the proposed work. Kanjanawanishkul (2016) has given the survey of formation control.

For path planning of wheeled mobile robot (WMR), there are two major criteria as,

a Feasibility: find a path that causes arrival at a goal state, regardless of its efficiency.

b Optimality: find a feasible path that optimises performance in some carefully specified manner, in addition to arriving in a goal state.

In literature, various path planning algorithms like

a road map (visibility graph and Voronoi diagram)

b cell decomposition (exact cell decomposition and approximate cell decomposition)

c potential field (the extended potential field method).

The motion control problem of WMRs can be addressed at three levels as

a geometric level, where the trajectory in the configuration space is designed in the presence of obstacles

b kinematic level, where the velocity profiles are designed

c dynamics level, where forces required by the robot are calculated such as in Charifa and Bikdash (2009) and Koren and Borenstein (1991).

A good robot motion planning method must lead to a robot trajectory with desirable geometrical features (e.g., robot moves to target along a short path while keeping a good safety distance from obstacles), desirable kinematical features (e.g., robot maintains a reasonably uniform and brisk speed while travelling but slows down in narrow spaces), and desirable dynamic features (viz. forces required by the robot are reasonable and easy to compute). The artificial potential field (APF) approach is a widely adopted approach in the mobile robot navigation and control as it can address all the three levels either directly or indirectly such as in Charifa and Bikdash (2009).

The sliding mode control (SMC) approach has been recognised as a one of the effective control strategy to design robust controllers for nonlinear systems operating under uncertainties and external disturbance conditions. SMC is well known for its inherent properties such that it is robust and insensitive to parametric uncertainties and disturbances (Utkin, 1992). SMC operation involves two phases viz. reaching phase and sliding phase. Once the system dynamics is brought on the sliding surface, the system leaves its own dynamics and follows the dynamics of sliding surface. Thus, it can not only stabilise the system but also can provide desired tracking dynamics. Since, WMR is a non-holonomic system with nonlinear dynamics, use of its linear model for controller design adds parametric uncertainty. Hence, to have robustness properties SMC is the best possible control strategy. However, the drawback of SMC is its inherent undesirable feature chattering. Due to chattering in control input, it has been restricted in some 
applications, since chattering may not be acceptable by physical final control elements or actuators. Chattering effects may be eliminated or at least reduced by replacing signum function in the control input with smoother functions like saturation function (sat), hyperbolic tangent function (tanh) with boundary layer (Khandekar et al., 2013). In this work, while implementing the control law, sign function is replaced by sat function with boundary layer $\varepsilon$ to reduce the chattering. To preserve the robustness of SMC, the value of $\varepsilon$ should be small, in this work the value of $\varepsilon$ is chosen as 0.001 . In literature many examples can be seen where the sliding mode has the ability to handle the uncertainties and disturbances (Pan et al., 2015, 2016; Parvat and Patre, 2017; Tak et al., 2016; Yang et al., 2017). Mu et al. (2015) presents a trajectory tracking control scheme for a two-WMR using sliding mode techniques. Guo and Qian (2015) have proposed the formation control of multiple non-holonomic two-wheeled robots using terminal SMC. Chang et al. (2013) has presented formation control for multi-robot dynamic systems using adaptive fuzzy terminal sliding-mode techniques.

Various control strategies like, input-output linearisation (Desai et al., 1998), backstepping-based (Dierks and Jagannathan, 2007; Fierro and Lewis, 1996), graph theoretic approach (Hernández-Martínez and Aranda-Bricaire, 2011; Cao et al., 2011; Zhao et al., 2017), direct Lyapunov method kinematic control (Fierro et al., 2001), model predictive control (Manikonda, et al., 1999; Dunbar and Murray, 2002; Xiao et al., 2016; Ebel et al., 2017), adaptive vision-based (Wang et al., 2017), switching strategy (Li and Xiao, 2005) for the leader-follower formation control method have appeared in the literature. Wang (1991) developed a strategy for formations of mobile robots where individual position coordinates have been allotted to a particular robot to maintain a specific position in the group with respect to the leader and neighbour. An approach for trajectory tracking of a mobile-robots formation by using the theory of linear algebra and numerical methods is presented in Rosales et al. (2011). Chen and Baoli (2015) have addressed the formation control problem of a group of WMRs with a virtual robot. Chwa (2016) presents a robust distance-based tracking control method for two-wheeled differential drive mobile robots in the presence of kinematic disturbances. Chen and Wang (2005) have presented a review on the current control issues and strategies on a group of unmanned autonomous vehicles/robots formation. Kowdiki et al. (2012) implemented formation control using APFs with a kinematic approach. Sanhoury et al. (2013) has proposed a new synchronous control rule for multiple mobile robot trajectories tracking while maintaining time varying formation. A layered formation framework for the control and coordination of group of mobile robots are shown in Kuppan Chetty et al. (2011).

In the existing research work, it is assumed that the leader robot somehow knows its path of navigation (Luca et al., 2001; Scaglia et al., 2009, 2010). This fact is actually a bottleneck for the implementation of fully autonomous formation control operation of the mobile robot swarms in reality. In this paper, a novel autonomous leader-follower formation control scheme has been proposed for a group of differentially driven WMR where the navigation and path planning of the leader robot as well as the follower robots has been achieved autonomously by employing APF. Moreover, in the proposed scheme, a sliding mode augmented path tracking control system has been designed considering kinematics, dynamics and the non-holonomic constraints of the differentially driven WMR. This paper presents a strategy based on modified form of APF such that leader robot finds its path in the cluster of static and dynamic obstacles to overcome the 
bottleneck associated with autonomous navigation of the leader robot in the leader-follower formation control which has never been addressed in the literature.

Modified APF has been adopted as a tool for autonomous navigation of the leader robot because of its versatile nature and its various useful features like less computation complexity and ease of programming in real-time platform. Here, the orientation angle of the leader robot changes depending on the number of static obstacles placed in the environment, so that leader robot will adapt the optimum path to reach the goal position by the influence of APFs. Then the follower robots will plan their path in order to maintain the desired separation distance and the bearing angle. Thus, APF has been employed directly for the leader robot and indirectly for the follower robot for autonomous path planning of the group of WMRs in the leader-follower formation control framework. Therefore, the proposed approach has overcome the technical bottleneck due to the lack of any full-fledged path planning component of the leader robot in dynamic situations during autonomous navigation in unknown environments. Now, the next task is to track the path generated by the APF for the leader as well as the path generated for the followers to maintain the desired separation distance and the bearing angle. Therefore, the formation control problem now becomes a trajectory tracking problem. However, it is quite challenging for WMRs to track the path generated by the APF for the leader and the path generated for the follower properly due to the non-holonomic constraints (Lee, 2004; Ssebazza, 2011). To address the issue of tracking the path generated by the APF and the associated challenges of non-holonomic constraints and various nonlinearities and uncertainties in the dynamic model of WMR, a sliding mode augmented composite tracking controller has been designed after eliminating the non-holonomic constraints of the WMR. SMC strategy has chosen to overcome the ill-effects due to the nonlinearities, model uncertainties, parameter variations, and disturbances. Thus, it can achieve robust tracking performance in the face of nonlinearities introduced by the APF algorithm during the path planning stages. The stability and robust tracking performance of the proposed control strategy has been proved theoretically. The effectiveness of the proposed leader-follower formation control has been established by simulation studies. To the best of the knowledge of the authors, leader-follower formation control employing APF for the fully autonomous path planning of the leader and consequently for the followers, and tracking the path generated by a sliding mode augmented composite tracking controller after eliminating the non-holonomic constraints of the WMR has never been addressed in the literature.

The major contributions of this paper are:

1 Autonomous path planning of the leader robot has been achieved.

2 Trajectory generated from the autonomous path planning of the leader and the follower WMRs has been tracked by a novel sliding mode augmented composite tracking controller to maintain the desired formation during the motion of the group of WMR.

This paper is organised as follows. Section 2 describes the autonomous path planning of the leader robot, in Section 3; the autonomous leader-follower formation control framework (model) is given. Section 4 deals with the trajectory tracking where the details of the controller design are elaborated. In Section 5, simulation results are presented and finally, Section 6 concludes the paper. 


\section{Autonomous path planning of the leader robot}

In this work, a modified form of the APF described by Khatib (1986) has been implemented. Here, a WMR has been mathematically modelled as a moving particle inside an APF that is generated by superposing an attractive potential which pulls the robot to a goal configuration and a repulsive potential that pushes the robot away from obstacles (Khatib, 1986; Ge and Cui, 2002; Zang et al., 2010). As explained in Section 2, then the potential function has been modified for the generation of the reference path velocity of the leader robot employing the incremental motion planning approach exploiting the concept of vortex field (Luca and Oriolo, 1994). This incremental motion planning in the framework of APF ensures avoidance of local minima problem that often occurs in APF-based navigation of WMR (Luca and Oriolo, 1994).

A potential function is a differentiable real valued function $U: \Re^{m} \rightarrow \mathfrak{R}$. The value of a potential function can be viewed as energy and hence the gradient of the potential is force. We use the gradient to define vector field, which assigns a vector to each point on the manifold. A gradient vector field assigns the gradient of some function to each point. The negative gradient of the generated global potential field is interpreted as an artificial force acting on the robot and dictating its motion.

Figure 1 Moving direction of robot in APF (see online version for colours)

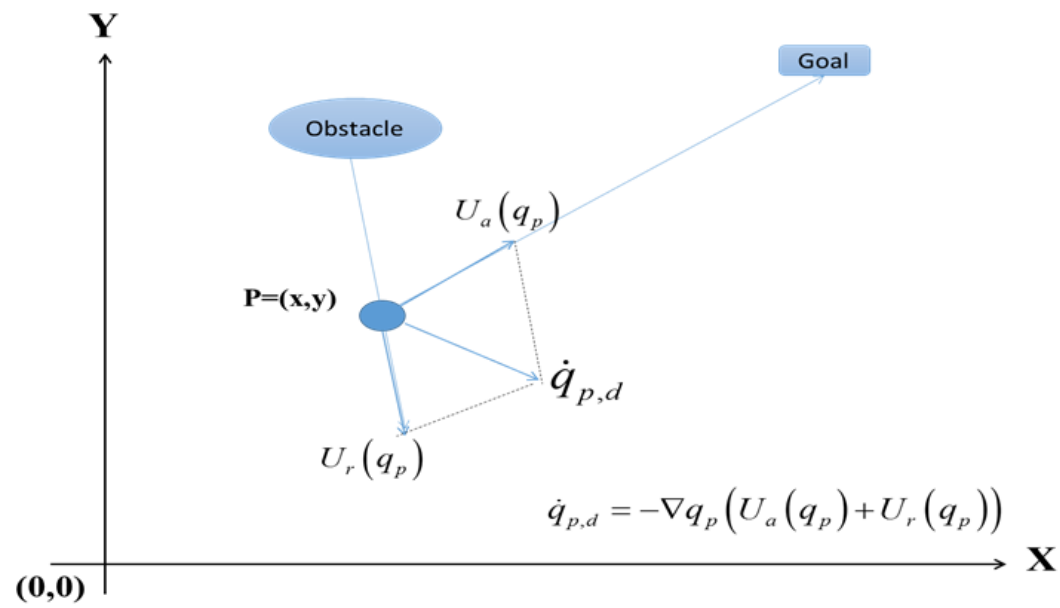

Assumption 1: It has been assumed that the group of WMR is operating on the horizontal plane.

Denoting by $q \in \mathfrak{R}^{n}$ the vector of generalised coordinates, assume that the system motion is subjected to a set of $m<n$ non-holonomic constraints, i.e., 'rolling without slipping' condition on the wheels, in the following form:

$$
A(q) \dot{q}=0
$$

Since constraint (1), involving time derivatives of the generalised coordinates, it is not integrable; the dimension of the configuration space cannot be reduced. However, all feasible velocities $\dot{q}$ should satisfy the following equation: 


$$
\dot{q}=S(q) v(t) ; v(t) \in \mathfrak{R}^{n-m}
$$

where $n-m$ independent columns of $S(q)$ are a basis for the null space of $A(q)$. Equation (2) is the kinematic model of the mobile robot.

We assume that control inputs are at the velocity level. Given any desired trajectory $\dot{q}_{d}(t)$, straight forward approach is to design the input command $v(t)$ using pseudo inversion.

$$
v(t)=S^{\#}(q) \dot{q}_{d}=\left[S^{T}(q) S(q)\right]^{-1} S^{T}(q) \dot{q}_{d}
$$

This solution locally minimises the error $\left(\dot{q}_{d}-\dot{q}\right)$ in a least square sense.

Since we are using APFs to drive the robot, hence

$$
\dot{q}_{d}=-\nabla q\left(U_{a}(q)+U_{r}(q)\right)
$$

With attractive potential $U_{a}(q)$ generated towards the goal $q_{g}$ and repulsive potential $U_{r}(q)$ generated by the obstacles.

In view of the planar nature of the motion problem, one can partition $q$ as $\left(q_{p}, q_{\theta}\right)$, with the positional part $q_{p}=(x, y) \in \mathfrak{R}^{2}$ and the angular part $q_{\theta} \in \mathfrak{R}^{n-2}$. Potential fields can then be set up for $q_{p}$, i.e., directly in the operational space where obstacles exist, by defining several Cartesian points $P_{i}=\left(x_{i}, y_{i}\right)$ on the mobile robot. Each of these control points will be subject to a field $U_{a, i}+U_{\text {rep }}$, being $U_{a, i}$ the attractive field associated to the goal for $P_{i}$. Then, the desired motion becomes

$$
\dot{q}_{d}=-\sum_{i} J_{i}^{T}(q) \nabla q_{p}\left(U_{a, i}\left(P_{i}\right)+U_{r}\left(P_{i}\right)\right)
$$

where $J_{i}(q)$ is the Jacobian of the kinematic mapping $P_{i}=f_{i}(q)$ of the $i^{\text {th }}$ control point. In order to allow for more flexibility in the design, one can then keep the positional part $\dot{q}_{p, d}$ of equation (5) and specify the desired motion of the angular part in a more general form

$$
\dot{q}_{\theta, d}=\Phi\left(q, \dot{q}_{p, d}\right)
$$

where an explicit dependence of the angular planning on the positional one has been introduced.

We apply the proposed approach to the kinematics of a differential WMR, where $q=(x, y, \theta)$ is the configuration vector. In this case, there is only one non-holonomic rolling constraint of the form (1):

$$
\left[\begin{array}{lll}
\sin \theta & -\cos \theta & 0
\end{array}\right]\left[\begin{array}{c}
\dot{x} \\
\dot{y} \\
\dot{\theta}
\end{array}\right]=0
$$

The kinematic model is as in equation (1). According to equation (3), the control input is chosen as 


$$
v(t)=S^{\#}(q) \dot{q}_{d}=\left[\begin{array}{ccc}
\cos \theta & \sin \theta & 0 \\
0 & 0 & 1
\end{array}\right]\left[\begin{array}{c}
\dot{x}_{d} \\
\dot{y}_{d} \\
\dot{\theta}_{d}
\end{array}\right]
$$

This expression has a direct geometric interpretation. The driving velocity $v$ is the orthogonal projection of the desired Cartesian velocity along the robot main axis, while the steering velocity $w$ exactly realises the desired rotation.

The positional part $\dot{q}_{p, d}=\left(\dot{x}_{d}, \dot{y}_{d}\right)$ is then obtained from equation (5) as

$$
\dot{q}_{p, d}=-\nabla q_{p}\left(U_{a}\left(q_{p}\right)+U_{r}\left(q_{p}\right)\right)
$$

To complete the planning method we assign the rotational part of $\dot{q}_{\theta, d}=\theta_{d}$ by specifying the form of $\Phi$ in equation (6) for the differential WMR it is convenient to use

$$
\dot{\theta}_{d}=a \tan 2\left\{\dot{x}_{d}, \dot{y}_{d}\right\}-\theta
$$

Since the differential WMR can instantaneously execute linear motions along its main axis, we force the robot to align with the field flow. The resulting command will be

$$
\begin{aligned}
& v=k_{p}\left(\dot{x}_{d} \cos \theta+\dot{y}_{d} \sin \theta\right) \\
& w=k_{\theta}\left(a \tan 2\left\{\dot{x}_{d}, \dot{y}_{d}\right\}-\theta\right)
\end{aligned}
$$

where $k_{p}$ and $k_{\theta}$ are the gains.

Figure 2 Geometric representation of motion of the robot in APF (see online version for colours)

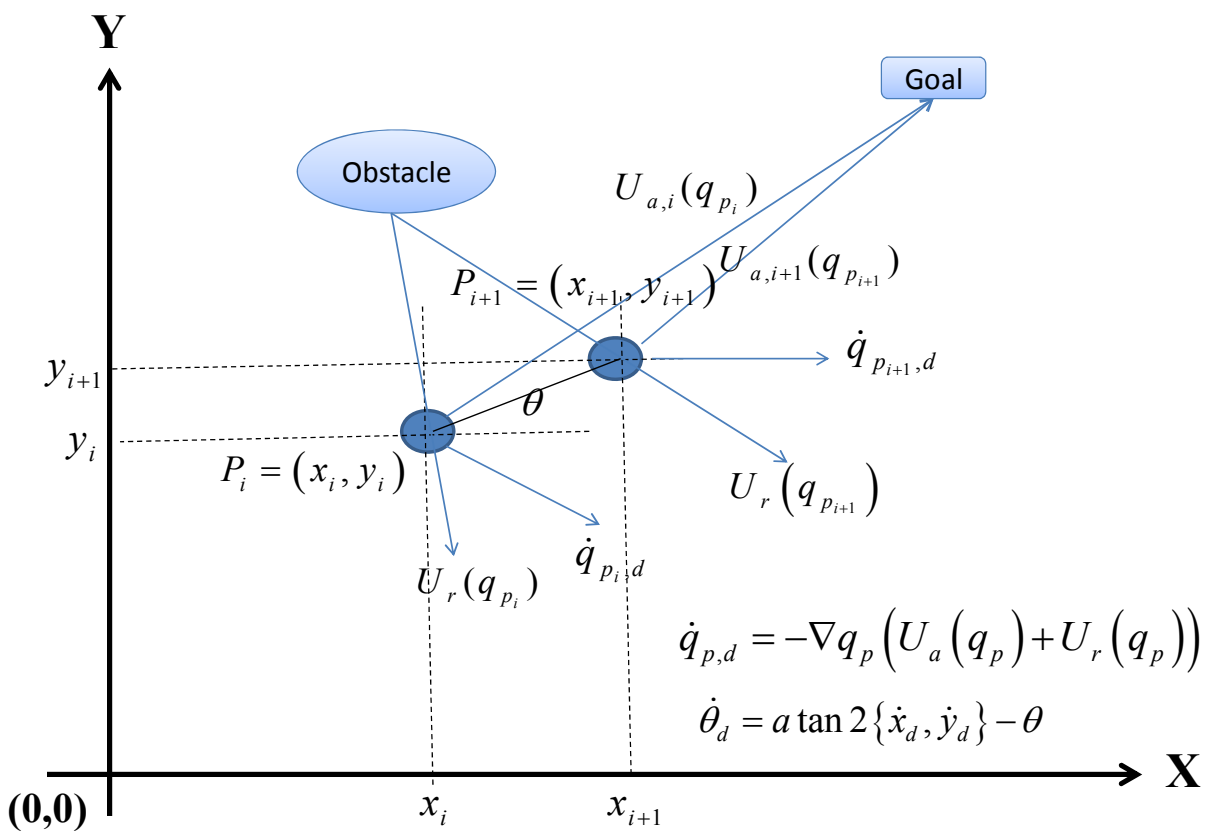


Figure 2 shows the analytical representation of how the position of the mobile robot at the next time step can be determined when it is navigating within the APF. From the above analytical interpretation, we can get the desired positional part $\dot{q}_{p, d}=\left(\dot{x}_{d}, \dot{y}_{d}\right)$ of the leader robot for the next time step, and the rotational part of the leader robot is $\dot{q}_{\theta, d}=\dot{\theta}_{d}$. Therefore, we are having the desired $\left(\dot{x}_{d}, \dot{y}_{d}, \dot{\theta}_{d}\right)$ of the leader robot for the next time step in discrete form.

By reducing the time step to the very small value, the desired or reference positional part and rotational part $\left(\dot{x}_{d}, \dot{y}_{d}, \dot{\theta}_{d}\right)$ of the leader robot can be approximated to as a continuous one as shown in the subsequent sections. The discrete form is useful for computer simulation; however, for the purpose of mathematical analysis and synthesis we have adopted the continuous time representation of the desired or reference position and orientation of the leader robot.

\section{Autonomous leader-follower formation control framework}

In this paper, the $l-\psi$ formation scheme has been considered because such scheme is applicable to all formations in which each robot has one leader except for the leading robot and we have assumed that there will be one leader robot and others will be the follower robots (Desai et al., 1998, 2001). In the $l-\psi$ control of the two mobile robots, the aim is to maintain a desired length (separation distance), $l_{12}^{d}$ and a desired relative angle (also known as bearing angle) $\psi_{12}^{d}$ between the two robots. The schematic diagram of the $l-\psi$ formation control of two mobile robots is shown in Figure 3.

Figure 3 The $l-\psi$ formation control scheme of two mobile robots (see online version for colours)

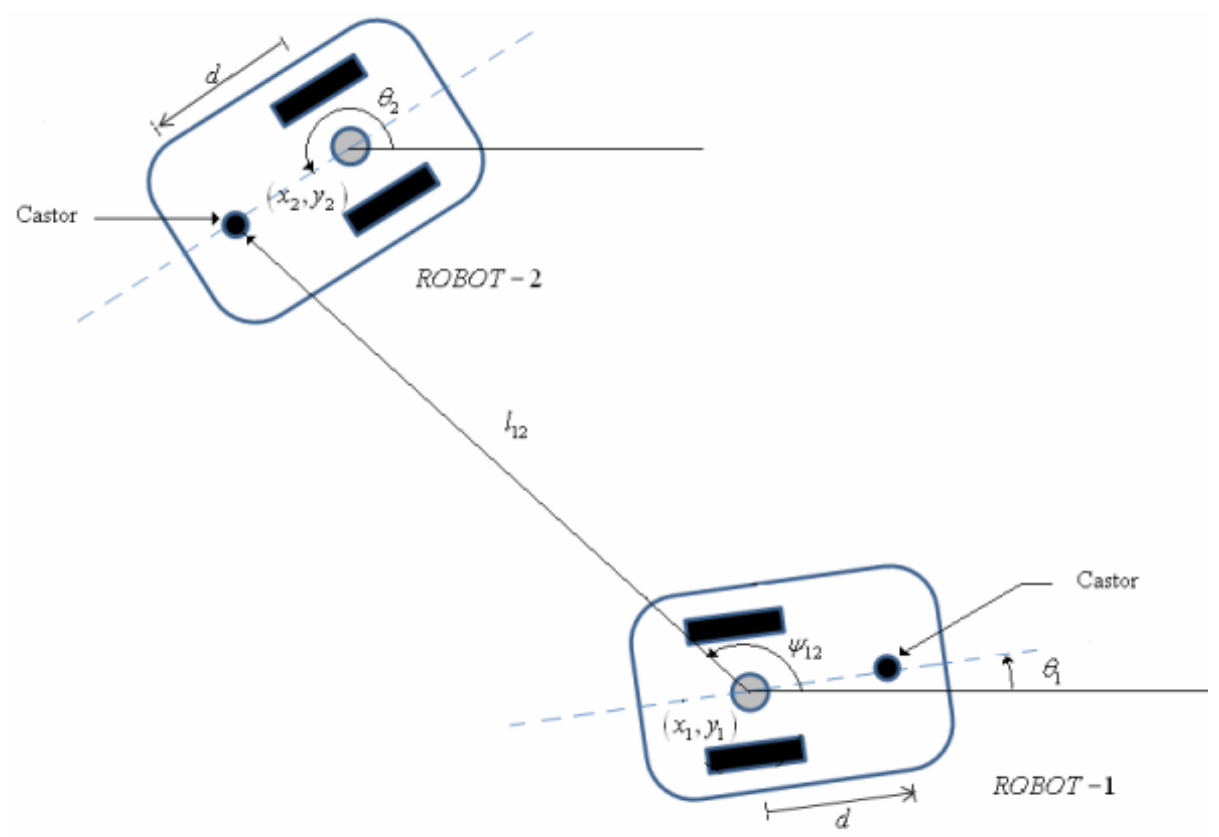


Referring to Figure 3 we can write the kinematic model of the leader and follower WMRs as following:

$$
\begin{aligned}
& \dot{x}_{i}=v_{i} \cos \theta_{i} \\
& \dot{y}_{i}=v_{i} \sin \theta_{i} \\
& \dot{\theta}_{i}=\omega_{i}
\end{aligned}
$$

where the subscript $i=1$ refers the leader WMR and for $i \geq 1$ refer to the follower WMRs, $\left(x_{i}, y_{i}\right)$ is the Cartesian coordinates of the leader or follower WMRs. Now, the kinematic model for leader-follower formation using the $l-\psi$ formation scheme can be written as:

$$
\begin{aligned}
& \dot{l}_{12}=v_{2} \cos \gamma_{1}-v_{1} \cos \psi_{12}+d \omega_{2} \sin \gamma_{1} \\
& \dot{\psi}_{12}=\frac{1}{l_{12}}\left\{v_{1} \sin \psi_{12}-v_{2} \sin \gamma_{1}+d \omega_{2} \cos \gamma_{1}-l_{12} \omega_{1}\right\} \\
& \dot{\theta}_{2}=\omega_{2}
\end{aligned}
$$

where $\gamma_{1}=\theta_{1}+\psi_{12}-\theta_{2}$.

In order to avoid collisions between the WMRs, $\mathrm{d}$ is the distance between the castor wheel and the centre of rear wheels and $l_{12}$ is the length of separation. Hence if two robots rotate simultaneously and the condition is $l_{12}>d$ not satisfied, then is a chance of collision. Hence, in order to avoid collision between the WMRs, it is essential to satisfy the condition $l_{12}>d$.

The desired separation distance $l_{12}^{d}$ and the desired relative angle (bearing angle) $\psi_{12}^{d}$ between the two robots are defined by:

$$
\begin{aligned}
& l_{12}^{d} x=x_{1}-d \cos \theta_{1}-x_{2} \\
& l_{12}^{d} y=y_{1}-d \sin \theta_{1}-y_{2} \\
& l_{12}^{d}=\sqrt{\left(l_{12}^{d} x\right)^{2}+\left(l_{12}^{d} y\right)^{2}} \\
& \psi_{12}^{d}=\tan ^{-1}\left(\frac{l_{12}^{d} y}{l_{12}^{d} x}\right)-\theta_{1}+\pi
\end{aligned}
$$

where $l_{12}^{d} x$ and $l_{12}^{d} y$ are the $x$ component and $y$ component of $l_{12}^{d}$.

Now, generalising for $n$ number of leaders and $m$ number of followers, equation (13) can be written as:

$$
\begin{aligned}
& \dot{l}_{i j}=v_{j} \cos \gamma_{1}-v_{i} \cos \psi_{i j}+d \omega_{j} \sin \gamma_{1} \\
& \dot{\psi}_{i j}=\frac{1}{l_{i j}}\left\{v_{i} \sin \psi_{i j}-v_{j} \sin \gamma_{1}+d \omega_{j} \cos \gamma_{1}-l_{i j} \omega_{i}\right\} \\
& \dot{\theta}_{j}=\omega_{j}
\end{aligned}
$$

where $\gamma_{1}=\theta_{i}+\psi_{i j}-\theta_{j}, i=1,2, \ldots, n$ and $j=1,2, \ldots, m$. There can be $n$ number of leaders and $m$ number of followers for each leader. In this work, one leader and two followers have been considered for simplifying the mathematical derivations. However, 
these mathematical derivations are equally valid for $n$ number of leaders and $m$ number of followers for each leader.

\section{$4 \quad$ Trajectory tracking}

The main objective in mobile robot trajectory tracking control is that the mobile robot should reach the Cartesian position $(x, y)$ with a pre-established orientation $\theta$ for each sampling period (Luca et al., 2001; Scaglia et al., 2009, 2010). In order to achieve this objective, only two control variables are available: the linear velocity $v$ and angular velocity $w$ of the robot. In this work, the leader's path is dictated by the APFs, and the orientation may change as per the static obstacles and dynamic obstacles in the environment, and the follower robots will track the leader's path by maintaining the desired separation distance $l_{12}^{d}$ and the bearing angle $\psi_{12}^{d}$.

Assumption 2: It has been assumed that the follower robots can communicate with the leader robot to get the position $(x, y)$ and orientation $\theta$ of the leader robot; so that they can maintain desired separation distance and relative bearing angle and the follower robots are also capable of avoiding collision with the obstacles and other robots.

\subsection{Design of tracking controller from kinematic considerations}

Kinematic control of differentially driven WMR is quite attractive from the practical point of view. This is because of the fact that the wheel-velocity control is commonly implemented on micro-controller-based computing platform and the reference velocity command comes from high level computer employed for path-planning operation and serves the current control objectives, like obstacle avoidance, minimum travel time, minimum energy consumption, etc. (Blažič, 2011).

To avoid collisions between the leader and the followers, separation distances are measured from the back of the leader to the front of the follower, and the kinematic equations for the front of the $j^{\text {th }}$ follower robot can be written as:

$$
\dot{q}=\left[\begin{array}{c}
\dot{x}_{j} \\
\dot{y}_{j} \\
\dot{\theta}_{j}
\end{array}\right]=\left[\begin{array}{cc}
\cos \theta_{j} & -d \sin \theta_{j} \\
\sin \theta_{j} & -d \cos \theta_{j} \\
0 & 1
\end{array}\right]\left[\begin{array}{c}
v_{j} \\
\omega_{j}
\end{array}\right]=S_{j}\left(q_{j}\right) v_{j}
$$

where $S_{j}\left(q_{j}\right)$ is the Jacobian matrix, $v_{j}$ is the velocity vector and $d$ is the distance from the rear axle to the front of the robot.

Now, we can write the following error system model (Fierro and Lewis, 1996) from the reference generated for the leader WMR, and consequently for the follower WMRs, depending upon attraction and repulsion forces experienced by the leader WMR in the APF as:

$$
\left[\begin{array}{l}
e_{j 1} \\
e_{j 2} \\
e_{j 3}
\end{array}\right]=\left[\begin{array}{ccc}
\cos \theta_{j} & \sin \theta_{j} & 0 \\
-\sin \theta_{j} & \cos \theta_{j} & 0 \\
0 & 0 & 1
\end{array}\right]\left[\begin{array}{l}
x_{j r}-x_{j} \\
y_{j r}-y_{j} \\
\theta_{j r}-\theta_{j}
\end{array}\right]
$$




$$
\dot{x}_{j r}=v_{j r} \cos \theta_{j r}, \dot{y}_{j r}=v_{j r} \sin \theta_{j r}, \dot{\theta}_{j r}=\omega_{j r}, \dot{q}_{j r}=\left[\begin{array}{lll}
\dot{x}_{j r} & \dot{y}_{j r} & \dot{\theta}_{j r}
\end{array}\right]^{T}
$$

where $x_{j}, y_{j}$, and $\theta_{j}$ are actual position and orientation of the robot, and $x_{j r}, y_{j r}$, and $\theta_{j r}$ are the positions and orientation of the reference or leader robot $j$ depending upon attraction and repulsion forces experienced by the leader WMR in the APF.

Then the error rate can be written as:

$$
\left[\begin{array}{c}
\dot{e}_{j 1} \\
\dot{e}_{j 2} \\
\dot{e}_{j 3}
\end{array}\right]=v\left[\begin{array}{c}
-1 \\
0 \\
0
\end{array}\right]+w\left[\begin{array}{c}
e_{j 2} \\
-e_{j 1} \\
1
\end{array}\right]+\left[\begin{array}{c}
v_{r} \cos e_{j 3} \\
v_{r} \sin e_{j 3} \\
w_{r}
\end{array}\right]
$$

Following the pioneering works of Fierro and Lewis (1996) and Kanayama et al. (1990), the velocity control input for the kinematic model of WMR, as given in equation (10), to achieve stable tracking can be computed as following:

$$
v_{c}=\left[\begin{array}{c}
v_{r} \cos e_{j 3}+k_{1} e_{1} \\
w_{r}+k_{2} v_{r} e_{j 2}+k_{3} v_{r} \sin e_{j 3}
\end{array}\right]
$$

The necessary proof of stability of the control input of equation (20) can be found in Fierro and Lewis (1996) and Kanayama et al. (1990) in detail. Now, let the WMR $i$ is acting as the leader and the WMR $j$ is acting as the follower, and $\left(x_{j r}, y_{j r}\right)$ are defined as points at a distance $l_{i j d}$ and a desired angle $\Psi_{i j d}$ from the leader robot. Therefore, the basic tracking control problems can be extended to a formation control as follows:

$$
\begin{aligned}
& {\left[\begin{array}{c}
\dot{x}_{i} \\
\dot{y}_{i} \\
\dot{\theta}_{i}
\end{array}\right]=\left[\begin{array}{cc}
\cos \theta_{i} & -d \sin \theta_{i} \\
\sin \theta_{i} & d \cos \theta_{i} \\
0 & 1
\end{array}\right]\left[\begin{array}{c}
v_{i} \\
w_{i}
\end{array}\right]} \\
& x_{j}=x_{i}-d \cos \theta_{i}+l_{i j}^{d} \cos \left(\Psi_{i j}^{d}+\theta_{i}\right) \\
& y_{j}=y_{i}-d \sin \theta_{i}+l_{i j}^{d} \sin \left(\Psi_{i j}^{d}+\theta_{i}\right) \\
& \theta_{j}=\theta_{i}
\end{aligned}
$$

and

$$
v_{j}=\left[\left|v_{i}\right| \quad\left|\omega_{i}\right|\right]^{T}
$$

Then the actual position and orientation of the follower $j$ with respect to leader $i$ can be defined as:

$$
\begin{aligned}
& x_{j}=x_{i}-d \cos \theta_{i}+l_{i j} \cos \left(\Psi_{i j}+\theta_{i}\right) \\
& y_{j}=y_{i}-d \sin \theta_{i}+l_{i j} \sin \left(\Psi_{i j}+\theta_{i}\right) \\
& \theta_{j}=\theta_{j}
\end{aligned}
$$

where $l_{i j}$ and $\Psi_{i j}$ is the actual separation and bearing of the follower $j$. The velocity expressions of the WMRs in equation (20) and (23) would actually play the role of reference velocity that we have to achieve for successful formation control of a group of differentially driven of the WMRs in the Leader-Follower formation control framework. 
The main assumption behind the design of the velocity control input of the kinematic controller is that the operating velocities of the WMRs are always available and a perfect velocity tracking will be done. However, the velocity control inputs generated by the kinematic controller do not ensure a perfect velocity tracking due to various factors arising out of the dynamics associated with the motion of WMR like non-holonomic constraints, nonlinearities, coupling forces due to high speed operations, model uncertainties, parameter variations, and disturbances. Therefore, the kinematic control input should be augmented by some nonlinear robust control technique so that the robots can operate at high speed and mitigates the problem due to non-holonomic constraints, nonlinearities, coupling forces due to high speed operations, model uncertainties, parameter variations, and disturbances.

\subsection{Dynamics of non-holonomic WMRs}

This section deals with the dynamics of WMR and the non-holonomic constraints and their removal from the dynamic model for further design of dynamic model-based robust control input for trajectory tracking in the leader-follower formation control framework.

Mobile robot systems having an $n$-dimensional configuration space with generalised coordinates $\left(q_{0}, \ldots q_{n}\right)$ and are subjected to $m$ constraints can be described as (Fierro and Lewis, 1996):

$$
M(q) \ddot{q}+V_{m}(q, \dot{q}) \dot{q}+F(\dot{q})+G(q)+\tau_{d}=B(q) \tau-A^{T}(q) \lambda
$$

where $M(q) \in R^{n \times n}$ is symmetric positive definite inertia matrix, $V_{m}(q, \dot{q}) \in R^{n \times n}$ is the centripetal and coriolis matrix, $F(\dot{q}) \in R^{n \times 1}$ is the surface friction, $G(q) \in R^{n \times 1}$ is the gravity vector, $\tau_{d}$ is bounded unknown disturbances, including unstructured un-modelled dynamics, $B(q) \in R^{n \times r}$ is the input transformation matrix, $\tau \in R^{n \times 1}$ is the input vector, $A(q)$ $\in R^{m \times n}$ is the matrix associated with constraint forces and $\lambda \in R^{n \times 1}$ is the vector of constraint forces.

It is considered that all the kinematic equality constraints are independent of time, and can be expressed as:

$$
A(q) \dot{q}=0
$$

Let $S(q)$ is a full rank matrix $(n-m)$ formed by a set of smooth and linearly independent vector fields spanning the null space of $A(q)$, i.e.

$$
S^{T}(q) A^{T}(q)=0
$$

According to (26) and (27), it is possible to find an auxiliary vector time function $v(t) \in$ $R^{n-m}$ such that for all $t$, we have:

$$
\dot{q}=S(q) v(t)
$$

The non-holonomic constraint states that the robot can only move in the direction normal to the axis of the driving wheels (i.e., the mobile base satisfies the conditions of pure rolling and non-slipping). This can be written as:

$$
\dot{y} \cos \theta-\dot{x} \sin \theta-d \dot{\theta}=0
$$




$$
\begin{aligned}
& S(q)=\left[\begin{array}{cc}
\cos \theta & -d \sin \theta \\
\sin \theta & d \cos \theta \\
0 & 1
\end{array}\right] ; v=\left[\begin{array}{c}
v \\
\omega
\end{array}\right] \\
& {\left[\begin{array}{c}
\dot{x} \\
\dot{y} \\
\dot{\theta}
\end{array}\right]=\left[\begin{array}{cc}
\cos \theta & -d \sin \theta \\
\sin \theta & d \cos \theta \\
0 & 1
\end{array}\right]\left[\begin{array}{c}
v \\
\omega
\end{array}\right]}
\end{aligned}
$$

where $|v| \leq V_{\max }$ and $|\omega| \leq W_{\max } . V_{\max }$ and $W_{\max }$ are the maximum linear and angular velocities of the mobile robot. System (30) is called the steering system of the vehicle as in Fierro and Lewis (1996).

The Lagrange formalism is used to find the dynamic equations of the mobile robot. In this case $G(q)=0$, because the trajectory of the mobile base is restricted to the horizontal plane, i.e., since the system, cannot change its vertical position, its potential energy, $U$, remains constant. The kinetic energy $K E$ is given by:

$$
k_{E}^{i}=\frac{1}{2} m_{i} v_{i}^{T} v_{i}+\frac{1}{2} \omega_{i}^{T} I_{i} \omega_{i}, K_{E}=\sum_{i=1}^{n_{i}} k_{E}^{i}=\frac{1}{2} \dot{q}^{T} M(q) \dot{q}
$$

Now, the dynamical equations of the mobile can be expressed in the matrix form of equation (20), where:

$$
\begin{aligned}
& M(q)=\left[\begin{array}{ccc}
m & 0 & m d \sin \theta \\
0 & m & -m d \cos \theta \\
m d \sin \theta & -m d \cos \theta & I
\end{array}\right], V(q, \dot{q})=\left[\begin{array}{c}
m d \dot{\theta}^{2} \cos \theta \\
m d \dot{\theta}^{2} \sin \theta \\
0
\end{array}\right], \\
& G(q)=0, B(q)=\frac{1}{r}\left[\begin{array}{cc}
\cos \theta & \cos \theta \\
\sin \theta & \sin \theta \\
R & -R
\end{array}\right], \tau=\left[\begin{array}{c}
\tau_{r} \\
\tau_{l}
\end{array}\right], \quad A^{T}(q)=\left[\begin{array}{c}
-\sin \theta \\
\cos \theta \\
-d
\end{array}\right], \\
& \lambda=-m\left(\dot{x}_{c} \cos \theta+\dot{y}_{c} \sin \theta\right) \dot{\theta}
\end{aligned}
$$

where $m$ is the mass of the mobile robot, $I$ is the moment of inertia of the mobile robot about its centre, $2 R$ and $r$ are the distances between the two driven wheels and the radius of the wheel, respectively. The terms, $\tau_{r}$ and $\tau_{l}$ are the torque control inputs generated by the right and the left DC motor, respectively. The system (25) is now transformed into a more appropriate representation for controller design purpose. Differentiating equation (28) and substituting this result in equation (21) and then multiplying it by $S^{T}$, we can eliminate the constraint matrix $A^{T}(q) \lambda$. The complete equations of motion of the non-holonomic mobile platform are given by

$$
\begin{aligned}
& \dot{q}=S v, \\
& S^{T} M S \dot{v}+S^{T}\left(M \dot{S}+V_{m} S\right) v+\bar{F}+\bar{\tau}_{d}=S^{T} B \tau,
\end{aligned}
$$

where $v(t) \in R^{n-m}$ is a velocity vector. By appropriate definitions we can re-write equation (35) as follows: 


$$
\bar{M}(q) \dot{v}+\bar{V}_{m}(q, \dot{q}) v+\bar{F}(v)+\bar{\tau}_{d}=\bar{B} \tau
$$

where $\bar{M}(q) \in R^{r \times r}$ is a symmetric, positive definite inertia matrix, $\bar{V}_{m}(q, \dot{q}) \in R^{r \times r}$ is a centripetal and coriolis matrix, $\bar{F}(v) \in R^{r \times 1}$ is the surface friction, $\bar{\tau}_{d}$ denotes bounded unknown disturbances, including unstructured unmodelled dynamics, and $\bar{\tau} \in R^{r \times 1}$ is the input vector. The distance between the centre of mass and the coordinate centre of the mobile robot is assumed to be zero; therefore, the effect of $\bar{V}_{m}$ can be eliminated from (29). The remaining variables in (29) are defined as $\bar{M}(q)=\left[\begin{array}{cc}m & 0 \\ 0 & I\end{array}\right]$ and $\bar{B}(q)=\frac{1}{r}\left[\begin{array}{cc}1 & 1 \\ R & -R\end{array}\right]$. By considering the surface friction and the disturbance torque as the modelling uncertainties and disturbances, then the dynamic equation (35) of the simple model of the mobile robot, assuming all the uncertainties and disturbances are zero, becomes as shown in (Chen et al., 2009).

$$
\dot{v}(t)=E \tau)(t)
$$

where the system matrix $E$ is

$$
E=\bar{M}^{-1}(q) \bar{B}(q)=\frac{1}{m . r . l}\left[\begin{array}{cc}
I & I \\
R m & -R m
\end{array}\right]
$$

\subsection{Design of sliding mode tracking controller}

The velocity term in equations (28), (33) and (34) is considered to be the actual velocity needed to overcome the effects of non-holonomic constraint and the dynamic effects associated with the motion of the WMRs. However, the dynamic model in equation (35) parameters may contain uncertainties and may change over time. Therefore, kinematic controller has been augmented in this work by incorporating SMC action to minimise the effects of nonlinearities, model uncertainties, parameter variations, and disturbances. Here, we utilise the SMC method to design a dynamic tracking controller which let the actual velocities of the mobile robot converge to the control velocities generated from the kinematic controller.

Let us define the auxiliary velocity tracking error and its derivative as shown in Chen et al. (2009) as:

$$
\begin{aligned}
& e_{c}(t)=\left[e_{c 1}, e_{c 2}\right]^{T}=v_{c}(t)-v(t) \\
& \dot{e}_{c}(t)=\dot{v}_{c}(t)-\dot{v}(t)
\end{aligned}
$$

Since, the error is changing dynamically with respect to time, we have selected PI-type sliding surface as in (Castro et al., 2009).

$$
S(t)=\left[\begin{array}{c}
S_{1}(t) \\
s_{2}(t)
\end{array}\right]=e_{c}(t)+\beta \int_{0}^{t} e_{c}(\tau) d \tau
$$


where $\beta$ is the sliding-surface integral parameter and $\beta>0$. It is noted in (41) that once the system is on the sliding surface $S=0$, then, $e_{c}(t)=-\beta \int_{0}^{t} e_{c}(\tau) d \tau$; hence, the tracking error $e_{c}(\infty) \rightarrow 0$ as $\beta>0$. Therefore, it is necessary to drive the closed-loop system toward the sliding surface $S=0$ to minimise the tracking error. Meanwhile, with the derivative $\dot{S}$ of sliding surface $S(t)$, one can obtain that.

$$
\dot{S}(t)=e_{c}(t)+\beta e_{c}(t)
$$

It is obvious that the tracking error $e_{c}(\infty) \rightarrow 0$ if the integral parameter $\beta$ is selected properly. From the concept of the equivalent control, the equivalent control law $\tau_{e q}$ is obtained by recognising that $\dot{S}(t)=0$ is a necessary condition for the state trajectory to stay on the sliding surface as explained in Slotine and Li (1991, pp.283-289). Thus, substituting (36) for (41), one can obtain.

$$
\dot{S}(t)=\left[\dot{v}_{c}(t)-E \tau(t)+\beta e_{c}(t)=0\right]
$$

Therefore, the equivalent control law $\tau_{e q}$ is given as.

$$
\tau_{e q}=E^{-1}\left[\dot{v}_{c}(t)+\beta e_{c}(t)\right]
$$

where $E^{-1}$ is selected as $E^{-1}=-\frac{r}{2 R}\left[\begin{array}{cc}-R m & -I \\ -R m & I\end{array}\right]$ and the equivalent control law $\tau_{e q}$ can make the system state remain on the sliding surface if the dynamic model is known exactly. However, if there are model uncertainties, in order to satisfy the sliding condition, we must employ the discontinuous control law $\tau_{s w}$ in (43) as in Slotine and $\mathrm{Li}$ (1991, pp.283-289). Thus the control law is composed of equivalent control $\tau_{e q}$ and switching control $\tau_{s w}$, i.e.

$$
\tau=\tau_{e q}+\tau_{s w}=E^{-1}\left[\dot{v}_{c}(t)+\beta e_{c}(t)+k \operatorname{sgn}(S)\right]
$$

where $k=\left[\begin{array}{cc}k_{1} & 0 \\ 0 & k_{2}\end{array}\right]$ and $k_{i}$ is a positive constant, and $\operatorname{sgn}(S)=\left[\operatorname{sgn}\left(s_{1}\right), \operatorname{sgn}\left(s_{2}\right)\right]^{T}$.

Then the dynamic equation (36) in the presence of parameter uncertainties and external disturbances becomes

$$
\dot{v}(t)=E \tau(t)+d_{s}(t)=\bar{E} \tau(t)+\Delta E \tau(t)+d_{s}(t)
$$

where $\bar{E}$ is denoted as the nominal part of the system matrix $E$ introduced by WMR parameters $r, R, m$ and inertia $I$. The $\Delta E$ is denoted as the uncertainties of the system matrix $E$. The $d_{s}(t)$ is called the external disturbance vector. Suppose

$$
\delta(t)=\left[\begin{array}{l}
\delta_{1}(t) \\
\delta_{2}(t)
\end{array}\right]=\Delta E \tau(t)+d_{s}(t)
$$

Then the dynamic equation of WMR can be written as

$$
\dot{v}(t)=E \tau(t)+\delta(t)
$$


Hence, the control input can be rewritten as

$$
\tau=\tau_{e q}+\tau_{s w}=\bar{E}^{-1}\left[\dot{v}_{c}(t)+\beta e_{c}(t)+k \operatorname{sgn}(S)\right]
$$

Theorem 1: The error vectors (17) and the auxiliary velocity tracking error $e_{c}$ (44) of the simple dynamic model of the WMR (36) will asymptotically converge to the zero vectors, if the kinematic controller (20) and the sliding mode tracking controller (48) are used.

Proof: The Lyapunoy functions form is a well known theory that can be used to prove stability for nonlinear systems

Let us define the Lyapunov function candidate as:

$$
V=V_{1}+V_{2}
$$

where

$$
\begin{aligned}
& V_{1}\left(e_{j 1}, e_{j 2}, e_{j 3}\right)=\frac{1}{2}\left(e_{j 1}^{2}+e_{j 2}^{2}\right)+\frac{1-\cos e_{j 3}}{k_{2}} \\
& V_{2}(S)=\frac{1}{2} S^{2}
\end{aligned}
$$

Substituting (20) and (21) for the time derivative of $V_{1}$ in (50), we obtain.

$$
\dot{V}_{1}=-k_{1} e_{j 1}^{2}-\frac{k_{3} v_{r} \sin ^{2} e_{j 3}}{k_{2}} \leq 0
$$

Differentiating (51), we can obtain

$$
\dot{V}_{2}=S \dot{S}=S\left[\dot{v}_{c}(t)-\dot{v}(t)+\beta e_{c}(t)\right]
$$

Substituting (42) and (47) in (53), we get

$$
\begin{aligned}
& \dot{V}_{2}=S\left[\dot{v}_{c}(t)-\{E \tau(t)+\delta(t)\}+\beta e_{c}(t)\right] \\
& \quad=S\left[\dot{v}_{c}(t)-E\left(E^{-1}\left\{\dot{v}_{c}(t)+\beta e_{c}(t)+k \cdot \operatorname{sgn}(S)\right\}\right)+\delta(t)+\beta e_{c}(t)\right] \\
& \quad=S[-k \cdot \operatorname{sgn}(S)+\delta(t)] \\
& \quad=S\left[-k \frac{|S|}{S}+\delta(t)\right] \leq 0 \\
& \quad=-k|S|+S \delta(t) \leq 0 \\
& S \delta(t) \leq k|S| \\
& S \delta(t) \leq k S \\
& \therefore k \geq \delta(t)
\end{aligned}
$$

Equation (54) gives the stability condition imposed on SMC. Let $\delta_{\max }$ be the upper bound of $\delta(t)$ then at any time $t$, if $k>\delta_{\max }$ is chosen then equation (54) holds true and the SMC can handle the effects of uncertainty and disturbance. 
We can conclude that $\dot{V}$ is negative semi-definite if condition in equation (54) is satisfied. That is, the error vector in equation (17) and the sliding surface $S$ approach to zero. It is noted in (41) that once $S=0$, then $e_{c}(t)=-\beta \int_{0}^{t} e_{c}(\tau) d \tau$ and it is obvious that $e_{c}(\infty) \rightarrow 0$

As it is known, the discontinuous switching function $\operatorname{sgn}(S)$ causes chattering. Boundary layer control with saturation function is frequently utilised to eliminate chattering. To reduce the chattering in control input, use the saturation function $\operatorname{sat}(S, \varepsilon)$. Hence by replacing $\operatorname{sgn}(S)$ by $\operatorname{sat}(S, \varepsilon)$ in equation (48) we get

$$
\tau=\tau_{e q}+\tau_{s w}=\bar{E}^{-1}\left[\dot{v}_{c}(t)+\beta e_{c}(t)+k \cdot \operatorname{sat}(S, \varepsilon)\right]
$$

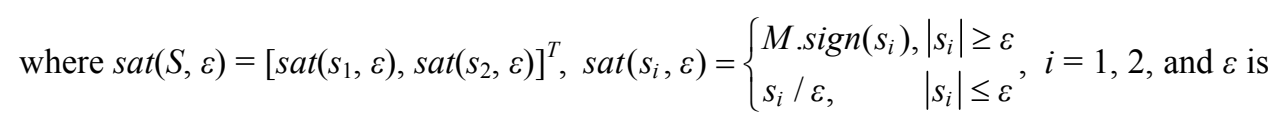
a small positive constant.

For $|s(t)| \geq \varepsilon, \operatorname{sat}(s)=\operatorname{sign}(s)$. However, in a small $\varepsilon$-vicinity of the origin, the so-called boundary layer, $\operatorname{sat}(s) \neq \operatorname{sign}(s)$ is continuous, with linear proportional feedback gain $\frac{M}{\varepsilon}$ within the boundary layer in the vicinity of the origin, $|s(t)| \leq \varepsilon$, and symmetrically saturated by $M$ for $s(t) \mid \geq \varepsilon$ outside the boundary layer (Guldner and Utkin, 2000).

\section{Simulation results}

A triangular formation of three identical mobile robots is considered where the leader's path of navigation is dictated by the APF and is considered as the desired formation trajectory. The simulation of the formation control with the proposed methodology law has been carried out in MATLAB (R2016a) with three obstacles in the environment. The initial position of the leader and two followers are defined as $(5,5),(5,3)$ and $(3,5)$ units respectively. The goal position of the leader robot is defined as $(25,25)$ units. The positions of the obstacles are defined as $(21,14),(12,12)$ and $(14,21)$ units. The leader's path is dictated by the APFs as described in section 2 is shown in red line in Figure 4(b). The path of the follower robots, while they chase the leader, is shown in blue lines. Robots positions after regular time intervals are also shown in Figure 4(b), these paths of leader and followers are the desired trajectory, and the controller designed will make the group of the mobile robots to track the desired trajectory.

The parameters selected for the mobile robot are as shown in Table 1.

Table 1 Parameters of wheeled mobile robot

\begin{tabular}{lc}
\hline Mass of the mobile robot (m) & $4 \mathrm{Kg}$ \\
Moment of inertia (I) & $2.5 \mathrm{Kg} \cdot \mathrm{m}^{2}$ \\
Distance between two wheels (2R) & $2 \times 0.15 \mathrm{~m}$ \\
Radius of the wheel (r) & $0.03 \mathrm{~m}$ \\
\hline
\end{tabular}


Figure 4 (a) Plot showing the changes in orientation of the leader robot while moving in the environment with obstacles (b) Plot showing the desired trajectories (c) Trajectory tracking of leader and followers (d) Velocity profile of leader robot (e) Convergence of error variables (f) Sliding surfaces $\mathrm{s}_{1}$ and $\mathrm{s}_{2}$ (g) Torques developed in the left wheel and right wheel DC motors, respectively (see online version for colours)

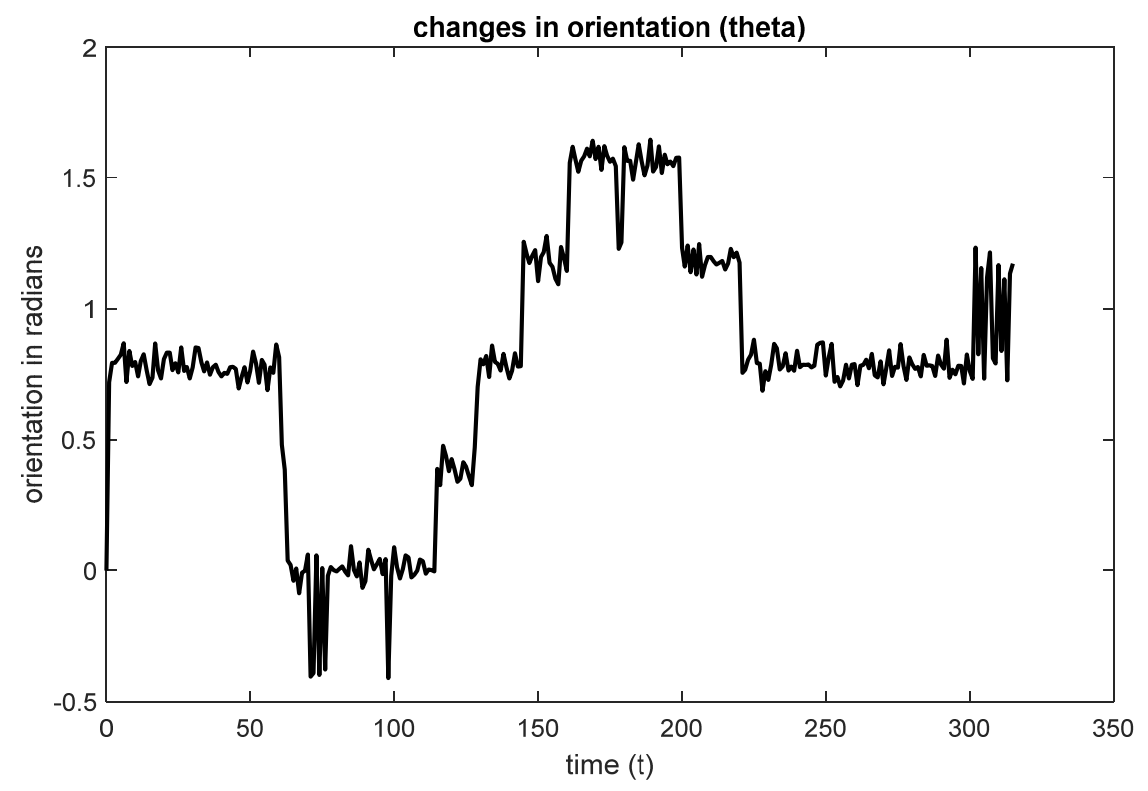

(a)

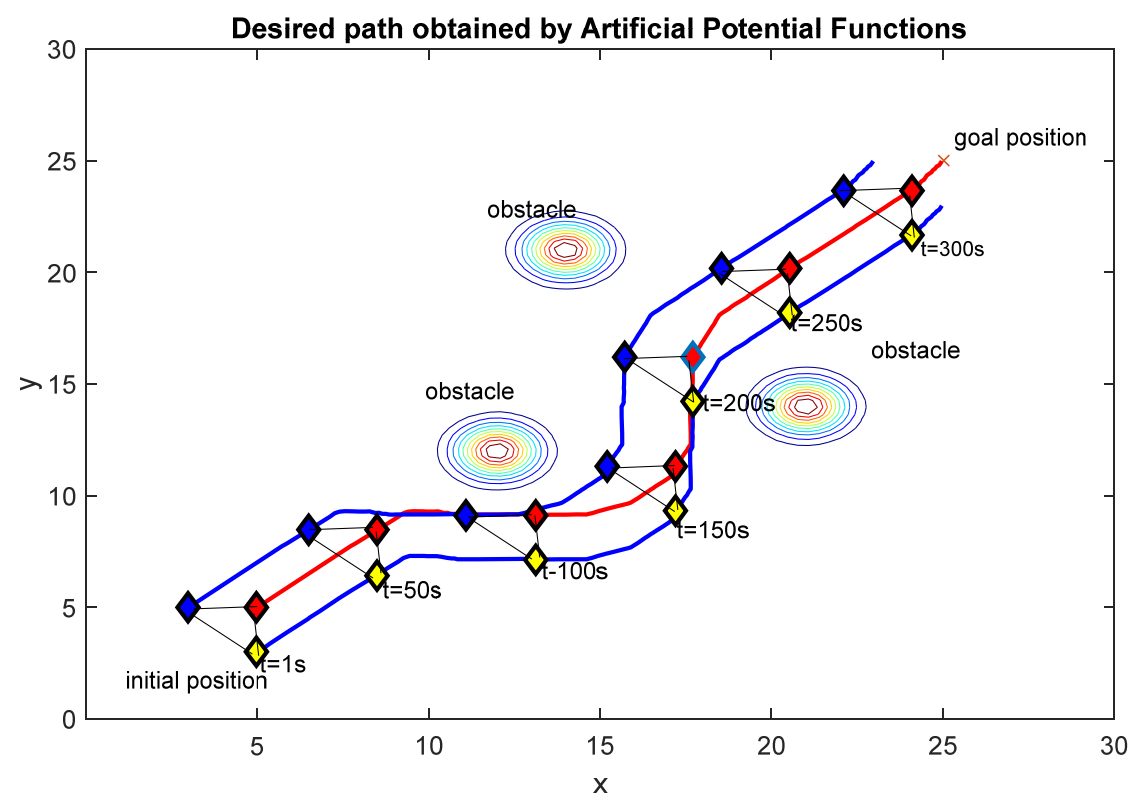

(b) 
Figure 4 (a) Plot showing the changes in orientation of the leader robot while moving in the environment with obstacles (b) Plot showing the desired trajectories (c) Trajectory tracking of leader and followers (d) Velocity profile of leader robot (e) Convergence of error variables (f) Sliding surfaces $\mathrm{s}_{1}$ and $\mathrm{s}_{2}$ (g) Torques developed in the left wheel and right wheel DC motors, respectively (continued) (see online version for colours)

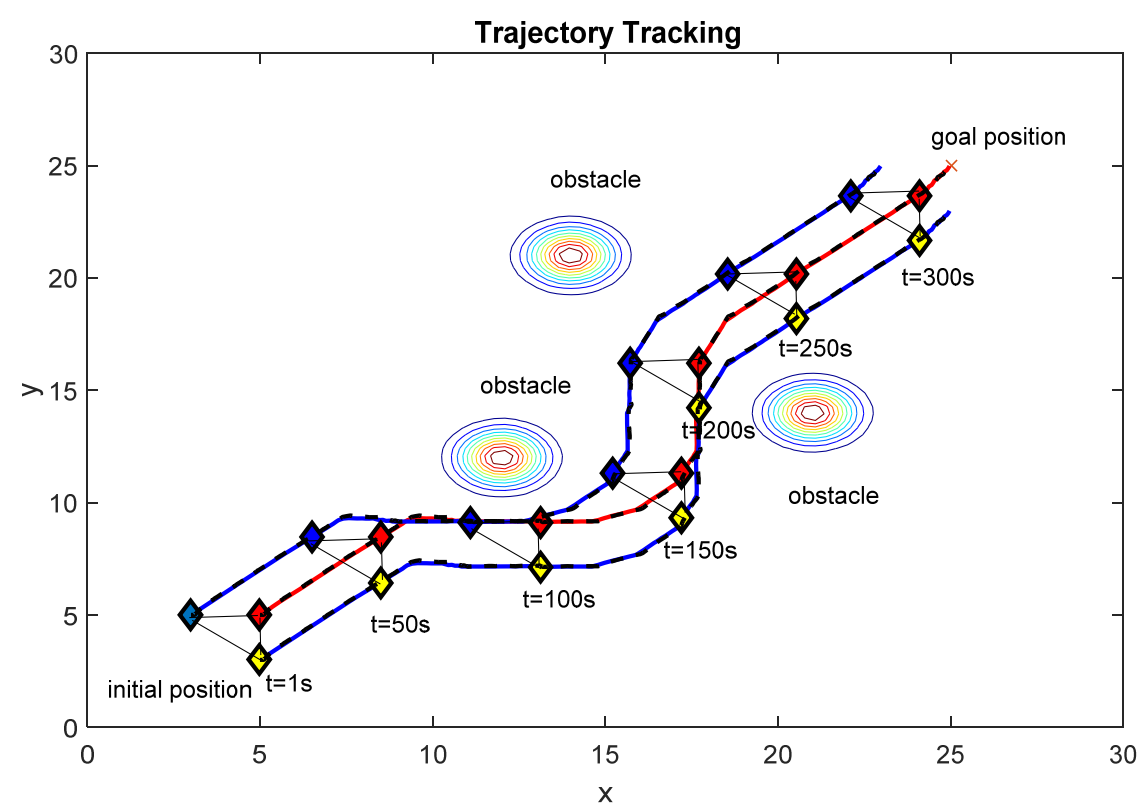

(c)

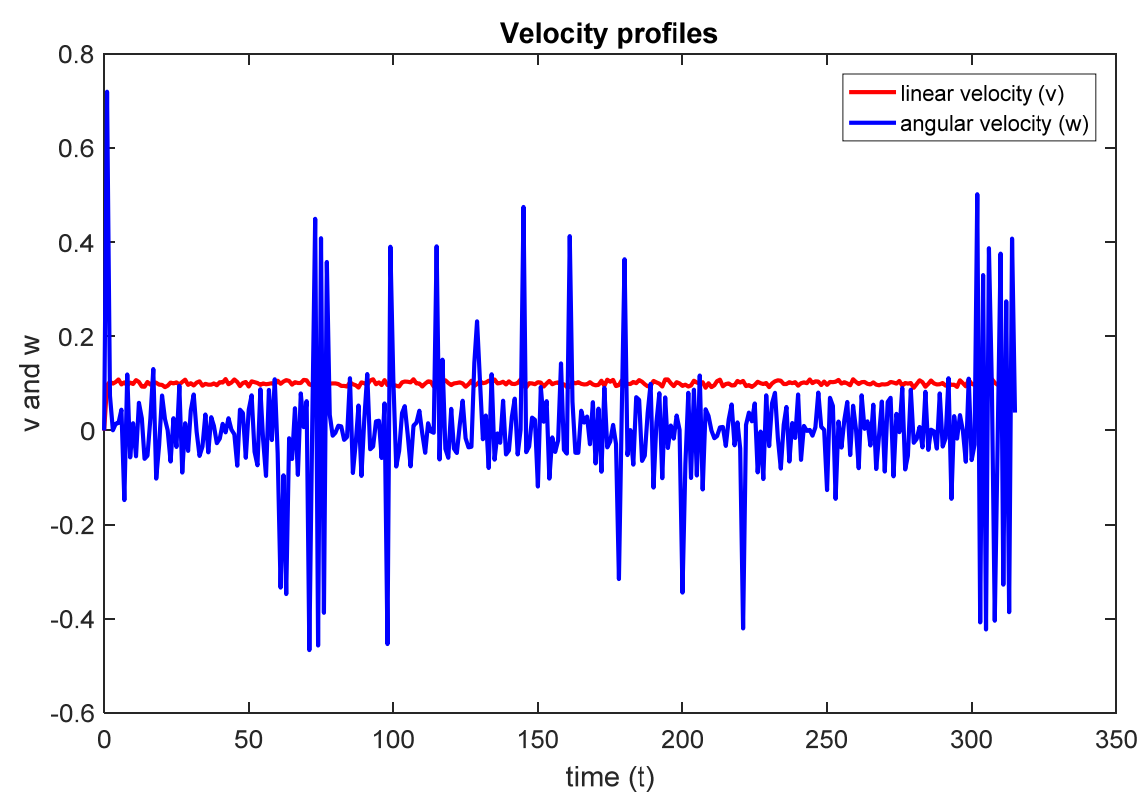

(d) 
Figure 4 (a) Plot showing the changes in orientation of the leader robot while moving in the environment with obstacles (b) Plot showing the desired trajectories (c) Trajectory tracking of leader and followers (d) Velocity profile of leader robot (e) Convergence of error variables (f) Sliding surfaces $\mathrm{s}_{1}$ and $\mathrm{s}_{2}$ (g) Torques developed in the left wheel and right wheel DC motors, respectively (continued) (see online version for colours)
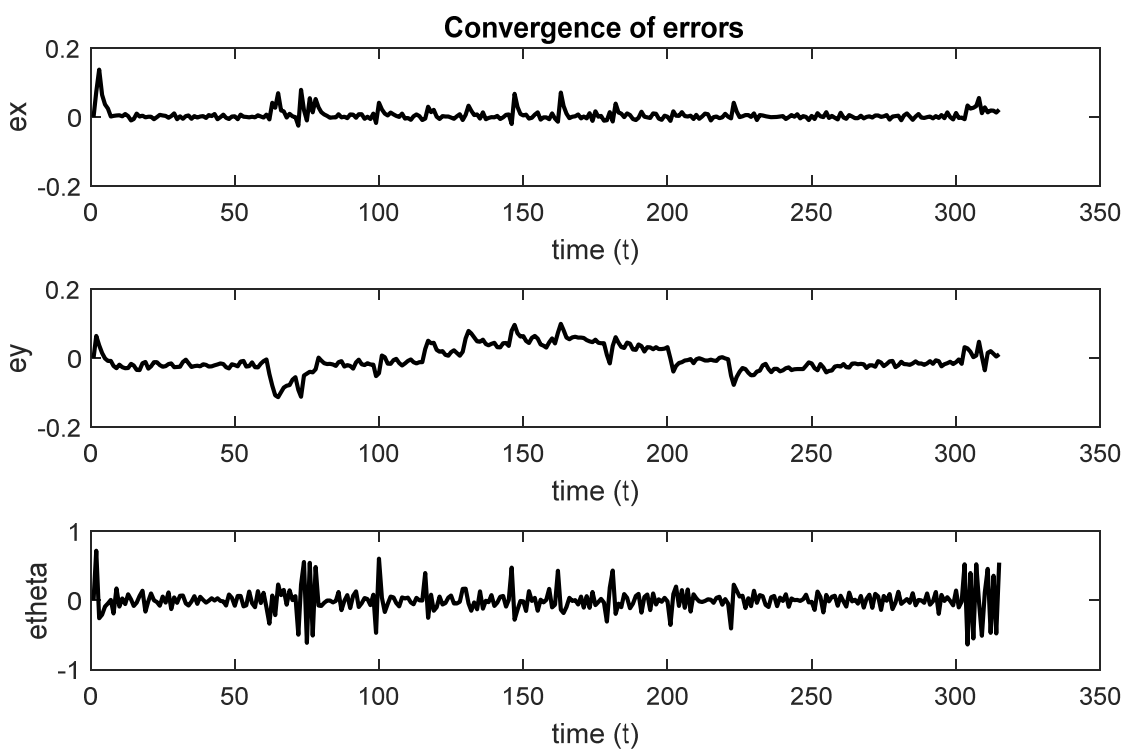

(e)
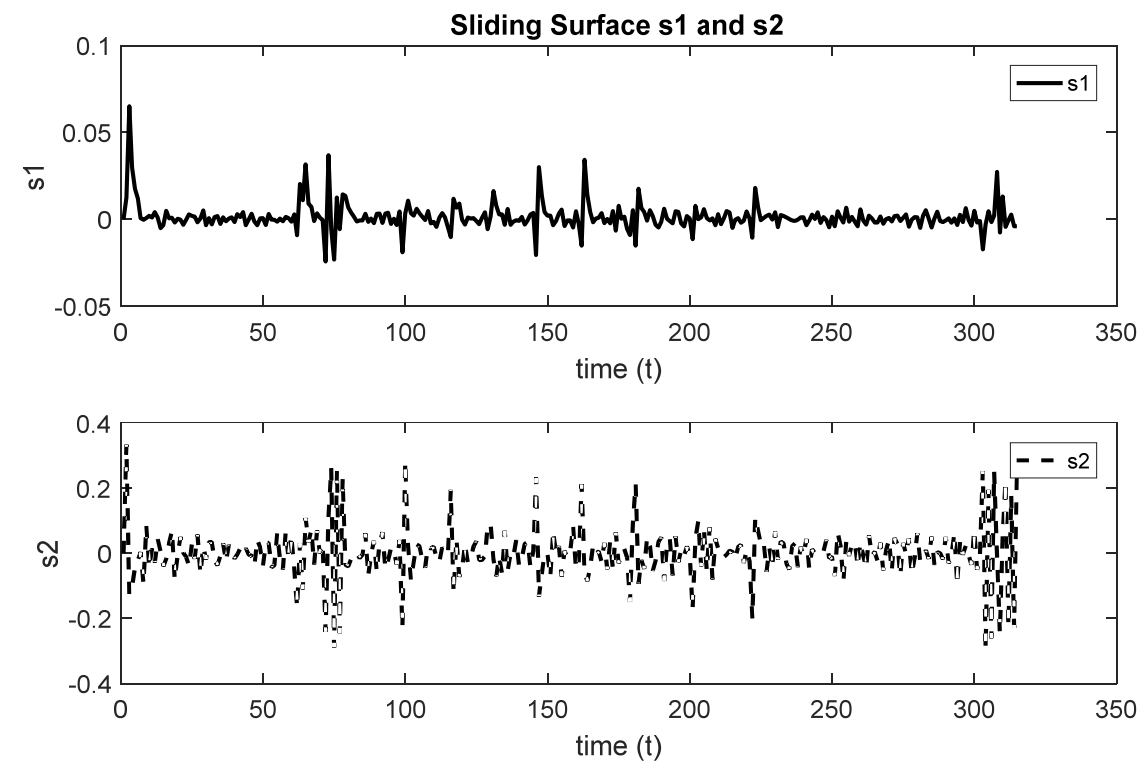

(f) 
Figure 4 (a) Plot showing the changes in orientation of the leader robot while moving in the environment with obstacles (b) Plot showing the desired trajectories (c) Trajectory tracking of leader and followers (d) Velocity profile of leader robot (e) Convergence of error variables (f) Sliding surfaces $\mathrm{s}_{1}$ and $\mathrm{s}_{2}$ (g) Torques developed in the left wheel and right wheel DC motors, respectively (continued) (see online version for colours)

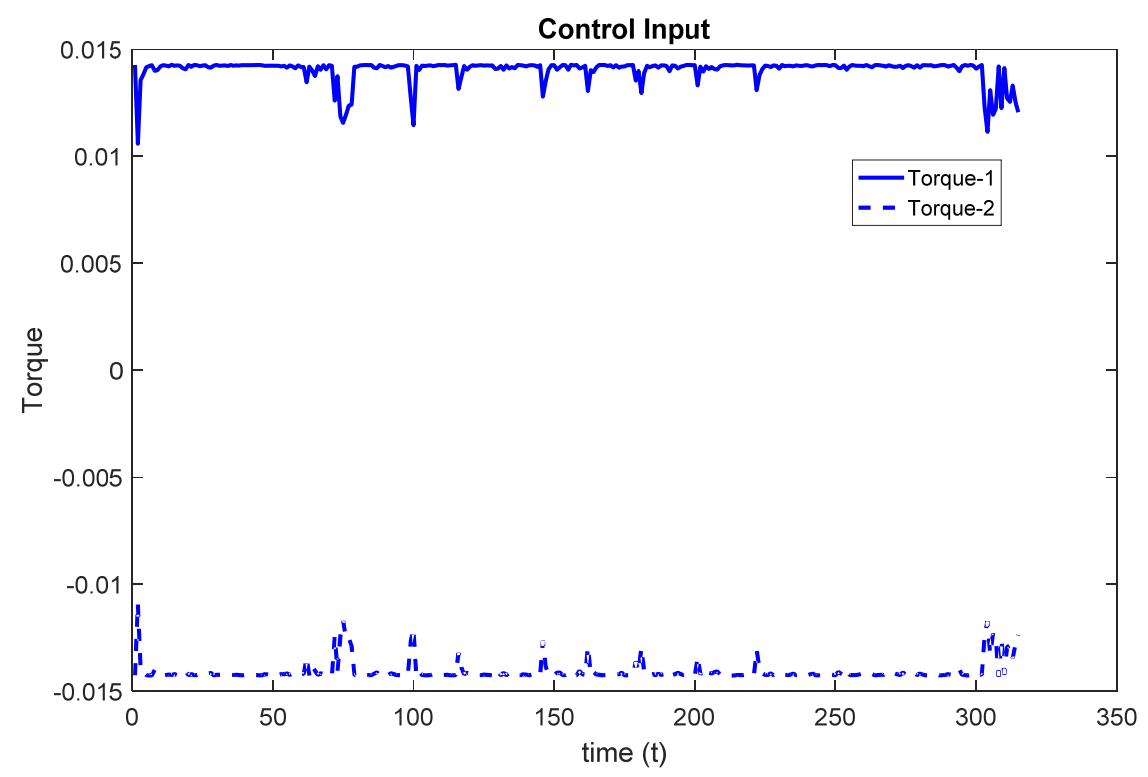

$(\mathrm{g})$

Based on the simulation results, it can be seen from Figure 4(b) that as the leader robot navigates itself by artificial potential field, its locomotion control is stable and robust against collision while reaching to the goal position and the followers are following the leader's path effectively. In Figure 4(a), we can see how the orientation of the leader robot is changing whenever there are obstacles in the environment. From Figure 4(c), it can be seen that the trajectory tracking by using kinematic control and augmented sliding mode controller is perfect (the actual path is overlapping the desired path). In case of the static obstacles the robots will move in the uniform speed, this can be seen in the velocity profile as shown in Figure 4(d) (the linear velocities $v$ are almost constant and the angular velocities $w$ are somewhat fluctuating, whenever there is change in the orientation of the WMR). Figure 4(e) shows the convergence of error of equation (18). Figure 4(f) shows how the sliding surface $S(t)$ of equation (41) are approaching towards zero vectors and Figure $4(\mathrm{~g})$ shows the torques developed in the left wheel and right wheel DC motors, respectively.

In order to get a greater insight into the tracking performance with this controller, a numerical analysis has also been carried out with the simulation data. Since, errors vary randomly and have positive and also negative values; root-mean-square error (RMSE) calculations are quite useful for assessing the tracking performance in contrast to the worst-case maximum and minimum errors. RMSE is defined as. 


$$
R M S E=\sqrt{\frac{\sum_{i=1}^{N}\left(e_{i}\right)^{2}}{N}}
$$

where $e_{i}=$ desired trajectory - actual trajectory

RMSE for $\mathrm{x}$-component

0.0989

RMSE for y-component

0.062

RMSE for theta - component 0.0064

The simulation of the formation control with the proposed methodology law has been carried out in MATLAB (R2016a) with three static obstacles and one dynamic obstacle in the environment. The initial position of the leader and two followers are defined as $(5,5),(5,3)$ and $(3,5)$ units respectively. The goal position of the leader robot is defined as $(25,25)$ units. The positions of the obstacles are defined as $(21,14),(12,12)$ and $(14$, $21)$ units. The initial position of the dynamic obstacle is defined as $(3,10)$ units. The simulation results for three static obstacles and one dynamic obstacle for two different speeds are shown in Figure 5(a) and Figure 5(b).

Figure 5 (a) Dynamic obstacle moving at slow speed (b) Dynamic obstacle moving with fast speed (see online version for colours)

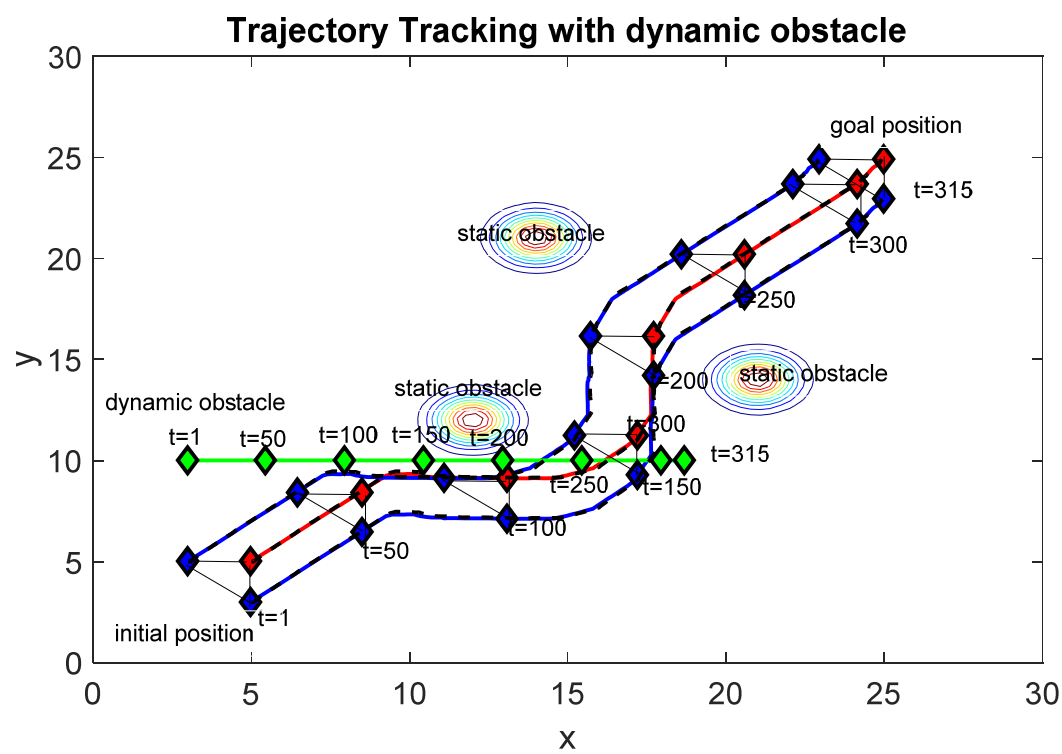

(a) 
Figure 5 (a) Dynamic obstacle moving at slow speed (b) Dynamic obstacle moving with fast speed (continued) (see online version for colours)

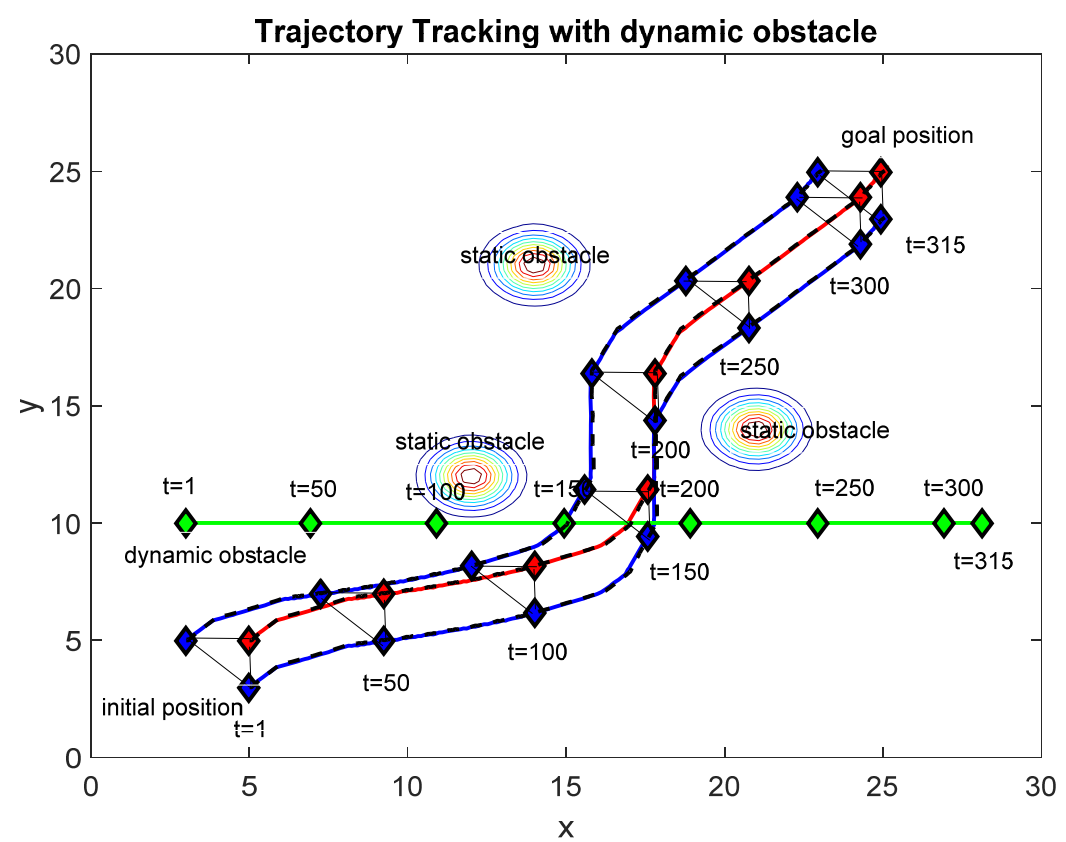

(b)

\section{Conclusions}

The main objective of this research is to develop a novel robust and autonomous formation control scheme of a group of differentially driven WMRs in the leader-follower formation control framework considering their non-holonomic constraints in an environment full of obstacles. To this end, autonomous path planning of the leader robot has been carried out as a function of reference velocity trajectory by employing an APF. The paths of the follower robots have been planned using the separation-bearing $l-\psi$ control in order to maintain a particular formation with respect to the leader robot. Then the resulting formation control problem has been treated as a path tracking problem. A sliding mode augmented tracking controller has been designed and applied for this tracking control. The closed-loop stability has been proved and the effectiveness of the proposed autonomous formation control scheme has been established in simulation studies.

\section{References}

Arai, T., Pagello, E. and Parker, L.E. (2002) 'Editorial: advances in multi-robot systems', IEEE Transactions on Robotics and Automation, Vol. 18, No. 5, pp.655-661.

Balch, T. and Arkin, R.C. (1998) 'Behavior-based formation control for multi-robot teams', IEEE Transactions on Robotics and Automation, Vol. 14, No. 6, pp.926-939. 
Blažič, S. (2011) 'A novel trajectory-tracking control law for wheeled mobile robots', Robotics and Autonomous Systems, Vol. 59, No. 11, pp.1001-1007.

Cao, K-C., Xiang, G. and Yang, H. (2011) 'Formation control of multiple nonholonomic mobile robots', International Conference on Information Science and Technology, pp.1038-1042.

Castro, R., Alvarez, J. and Martinez, J. (2009) 'Robot formation control using backstepping and sliding mode techniques', International Conference on Electrical Engineering, Computing Science and Automatic Control, pp.1-6.

Chang, Y.H., Yang, C.Y., Chan, W.S., Chang, C.W. and Tao, C.W. (2013) 'Leader-following formation control of multi-robot systems with adaptive fuzzy terminal sliding-mode controller', in 2013 International Conference on System Science and Engineering (ICSSE), July, pp.45-50, IEEE.

Charifa, S. and Bikdash, M. (2009) 'Comparison of geometrical, kinematic, and dynamic performance of several potential field methods', IEEE Conference SOUTHEASTCON '09, pp.18-23.

Chen, C., Li, T.S., Yeh, Y. and Chang, C. (2009) 'Design and implementation of an adaptive sliding-mode dynamic controller for wheeled mobile robots', Mechatronics, Vol. 19, No. pp.156-166.

Chen, L. and Baoli, M. (2015) 'A nonlinear formation control of wheeled mobile robots with virtual structure approach', Proceedings of the 34th Chinese Control Conference, Hangzhou, China, pp.3307-3312.

Chen, Y.Q. and Wang, Z. (2005) 'Formation control: a review and a new consideration', in 2005 IEEE/RSJ International Conference on Intelligent Robots and Systems, IROS 2005, August, pp.3181-3186, IEEE.

Chwa, D. (2016) 'Robust distance-based tracking control of wheeled mobile robots using vision sensors in the presence of kinematic disturbances', IEEE Transactions on Industrial Electronics, Vol. 63, No. 10, pp.6172-6183.

Desai, J.P., Kumar, V. and Ostrowski, J.P. (2001) 'Modeling and control of formations of nonholonomic mobile robots', IEEE Transactions on Robotics and Automation, Vol. 17, No. 6, pp.905-908.

Desai, J.P., Ostrowski, J.P. and Kumar, V. (1998) 'Controlling formations of multiple mobile robots', in Proceedings 1998 IEEE International Conference on Robotics and Automation, pp.2864-2869.

Dierks, T. and Jagannathan, S. (2007) 'Control of nonholonomic mobile robot formations: backstepping kinematics into dynamics', IEEE International Conference on Control Applications, pp.94-99.

Dunbar, W.B. and Murray, R.M. (2002) 'Model predictive control of coordinated multi-vehicle formations', in Proceedings of the 4lst IEEE Conference on Decision and Control, Vol. 4, pp.4631-4636, IEEE.

Ebel, H., Ardakani, E.S. and Eberhard, P. (2017) 'Distributed model predictive formation control with discretization-free path planning for transporting a load', Robotics and Autonomous Systems, Vol. 96, pp.211-223.

Egerstedt, M. and Hu, X. (2001) 'Formation constrained multi-agent control', IEEE Transactions on Robotics and Automation, Vol. 17, No. 6, pp.947-951.

Fierro, R. and Lewis, F.L. (1996) 'Control of a nonholonomic mobile robot: backstepping kinematics into dynamics', Proc. IEEE Conference on Decision Control, pp.1722-1727.

Fierro, R., Das, A.K., Kumar, V. and Ostrowski, J.P. (2001) 'Hybrid control of formations of robots', International Conference in Robotics and Automation, pp.157-162.

Ge, S.S. and Cui, Y.J. (2002) 'Dynamic motion planning for mobile robots using potential field method', Autonomous Robots, Vol. 13, No. 3, pp.207-222.

Guldner, J. and Utkin, V.I. (2000) 'The chattering problem in sliding mode systems', in 14th Int. Symp. Math. Theory Netw. Syst. (MTNS), Perpignan, France, Vol. 11. 
Guo, J. and Qian, D. (2015) 'Terminal sliding mode formation control of uncertain multiple robots', Int. J. of Advanced Mechatronic Systems, Vol. 6, Nos. 2/3, pp.118-127.

Hernández-Martínez, E.G. and Aranda-Bricaire, A. (2011) 'Convergence and collision avoidance in formation control: a survey of the artificial potential functions approach', Multi-Agent Systems - Modeling, Control, Programming, Simulations and Applications, Intech, London, UK, pp.103-126.

Kanayama, Y., Kimura, Y., Miyazaki, F. and Noguchi, T. (1990) 'A stable tracking control method for an autonomous mobile robot', Proc. IEEE International Conference on Robotics and Automation, pp.384-389.

Kanjanawanishkul, K. (2016) 'Formation control of mobile robots: survey', Journal of Engineering, Vol. 4, No. 1, pp.50-64.

Khandekar, A.A., Malwatkar, G.M. and Patre, B.M. (2013) 'Discrete sliding mode control for robust tracking of higher order delay time systems with experimental application', ISA Transactions, Vol. 52, No. 1, pp.36-44.

Khatib, O. (1986) 'Real-time obstacle avoidance for manipulators and mobile robots', The International Journal of Robotics Research, Vol. 5, No. 1, pp.90-98.

Koren, Y. and Borenstein, J. (1991) 'Potential field methods and their inherent limitations for mobile robot navigation', IEEE International Conference on Robotics and Automation, pp.1398-1404.

Kowdiki, K.H., Barai, R.K. and Bhattacharya, S. (2012) 'Leader-follower formation control using artificial potential functions: a kinematic approach', in 2012 International Conference on Advances in Engineering, Science and Management (ICAESM), March, pp.500-505, IEEE.

Kuppan Chetty, R.M., Singaperumal, M., Nagarajan, T. and Tetsunari, I. (2011) 'Coordination control of wheeled mobile robots - a hybrid approach', Int. J. of Computer Applications in Technology, Vol. 41, Nos. 3/4, pp.195-204.

Lee, L-F. (2004) Decentralized Motion Planning within an Artificial Potential Framework (APF) for Cooperative Payload Transport by Multi-Robot Collectives, Master's thesis, Department of Mechanical and Aerospace Engineering, State University of New York at Buffalo, Buffalo, New York 14260

Lewis, M.A. and Tan, K.H. (1997) 'High precision formation control of mobile robots using virtual structures', Autonomous Robots, Vol. 4, No. 4, pp.387-403.

Li, X. and Xiao, J. (2005) 'Robot formation control in leader-follower motion using direct Lyapunov method', International Journal of intelligent Control and Systems, Vol. 10, No. 3, pp.244-250.

Luca, A.D. and Oriolo, G. (1994) 'Local incremental planning for nonholonomic mobile robots', in 1994 IEEE International Conference on Robotics and Automation, Proceedings, pp.104-110, IEEE.

Luca, A.D., Oriolo, G. and Vendittelli, M. (2001) 'Control of wheeled mobile robots: an experimental overview', Lecture Notes in Control and Information Sciences, Vol. 270, pp.181-226, Springer, Berlin, Heidelberg.

Manikonda, V., Arambel, P.O., Gopinathan, M., Mehra, R.K. and Hadaegh, F.Y. (1999) 'A model predictive control-based approach for spacecraft formation keeping and attitude control', in American Control Conference, Proceedings of the 1999, Vol. 6, pp.4258-4262, IEEE.

$\mathrm{Mu}$, J., Yan, X-G., Spurgeon, S.K. and Mao, Z. (2015) 'Trajectory tracking control of a two wheeled mobile robot using sliding mode techniques', Proceedings of the 34th Chinese Control Conference, Hangzhou, China, pp.3307-3312.

Olfati-Saber, R., Fax, J.A. and Murray, R.M. (2007) 'Consensus and cooperation in networked multi-agent systems', Proceedings of the IEEE, Vol. 95, No. 1, pp.215-233.

Pan, H., Sun, W., Gao, H. and Jing, X. (2016) 'Disturbance observer-based adaptive tracking control with actuator saturation and its application', IEEE Transactions on Automation Science and Engineering, Vol. 13, No. 2, pp.868-875. 
Pan, H., Sun, W., Gao, H. and Yu, J. (2015) 'Finite-time stabilization for vehicle active suspension systems with hard constraints', IEEE Transactions on Intelligent Transportation Systems, Vol. 16, No. 5, pp.2663-2672.

Parvat, B.J. and Patre, B.M. (2017) 'Robust dynamic sliding mode control for a class of uncertain multi-variable process', in Proceedings of the International Conference on Data Engineering and Communication Technology, pp.67-76, Springer Singapore.

Ren, W. and Beard, R.W. (2004) 'Formation feedback control for multiple spacecraft via virtual structures', IEE Proceedings - Control Theory and Applications, Vol. 151, No. 3, pp.357-368.

Reynolds, C. (1987) 'Flocks, herds, and schools: a distributed behavioural model', Computer Graphics, July, Vol. 21, No. 4, pp.25-34.

Rosales, A., Scaglia, G., Mut, V. and Sciascio, F. (2011) 'Formation control and trajectory tracking of mobile robotic systems - a linear algebra approach', Robotica, Vol. 29, No. 3, pp.335-349.

Sanhoury, I.M.H., Amin, S.H.M. and Husain, A.R. (2013) 'Formation control of multiple mobile robots utilising synchronisation approach', Int. J. of Mechatronics and Manufacturing Systems, Vol. 6, No. 1, pp.94-114.

Scaglia, G., Quintero, L., Mut, V. and di Sciascio, F. (2009) 'Numerical methods based controller design for mobile robots', Robotica, Vol. 27, No. 02, pp.269-279.

Scaglia, G., Rosales, A., Quintero, O.L., Mut, V. and Agrawal, R. (2010) 'A linear-interpolation-based controller design for trajectory tracking of mobile robots', Control Engineering Practice, Vol. 18, No. 3, pp.318-329.

Slotine, J.J.E. and Li, W. (1991) Applied Nonlinear Control', pp.276-307, Prentice Hall Inc., New Jersey.

Ssebazza, L. (2011) DGPS-Based Localization for Path Following of Outdoor Wheeled Mobile Robots, Master's thesis, Department of Applied Science, Dalhousie University, Halifax, Nova Scotia.

Tak, R., Desai, J.V. and Rajpurohit, B.S. (2016) 'Sliding mode control: a novel control method for unstructured systems', in Intelligent Systems and Control (ISCO), pp.1-6.

Utkin, V.I. (1992) Sliding Modes in Optimization and Control Problems, Springer-Verlag Berlin, Heidelberg.

Wang, H., Guo, D., Liang, X., Chen, W., Hu, G. and Leang, K.K. (2017) 'Adaptive vision-based leader-follower formation control of mobile robots', IEEE Transactions on Industrial Electronics, Vol. 64, No. 4, pp.2893-2902.

Wang, P.K.C. (1991) 'Navigational strategies for multiple autonomous mobile robots moving in formation', Journal of Robotic Systems, Vol. 8, No. 2, pp.177-195.

Xiao, H., Li, Z. and Chen, C.P. (2016) 'Formation control of leader-follower mobile robots systems using model predictive control based on neural-dynamic optimization', IEEE Transactions on Industrial Electronics, Vol. 63, No. 9, pp.5752-5762.

Yang, Y., Zhong, W., Tan, S.C. and Hui, S.Y.R. (2017) 'Dynamic improvement of wireless power transfer systems with maximum energy efficiency tracking by sliding mode control', in 2017 IEEE 3rd International Future Energy Electronics Conference and ECCE Asia (IFEEC 2017-ECCE Asia), pp.1736-1740, IEEE.

Zang, M., Shen, Y. and Wang, Q. (2010) 'Dynamic artificial potential field based multi-robot formation control', IEEE International Conference Instrumentation and Measurement Technology, pp.1530-1534.

Zhao, Y.D., Kim, D.E., Yoon, H.N., Han, S.I. and Lee, J.M. (2017) 'Consensus formation control of multiple wheeled mobile robots', in 2017 IEEE 26th International Symposium on Industrial Electronics (ISIE), pp.1081-1086, IEEE. 